\title{
A Hybrid Model Integrating Singular Spectrum Analysis and Backpropagation Neural Network for Stock Price Forecasting
}

\author{
Asmaa Y. Fathi ${ }^{1 *}$, Ihab A. El-Khodary ${ }^{1}$, Muhammad Saafan ${ }^{2}$ \\ ${ }^{1}$ Department of Operations Research and Decision Support, Faculty of Computers and Information, Cairo University, Orman \\ 12613, Giza, Egypt \\ ${ }^{2}$ Department of Petroleum Engineering, Universiti Teknologi PETRONAS (UTP), Seri Iskandar 32610, Perak Darul Ridzuan, \\ Malaysia
}

Corresponding Author Email: a.fathi@fci-cu.edu.eg

https://doi.org/10.18280/ria.350606

Received: 29 September 2021

Accepted: 3 December 2021

\section{Keywords:}

stock market, stock price prediction, singular spectrum analysis, neural network, hybrid model

\begin{abstract}
The primary purpose of trading in stock markets is to profit from buying and selling listed stocks. However, numerous factors can influence the stock prices, such as the company's present financial situation, news, rumor, macroeconomics, psychological, economic, political, and geopolitical factors. Consequently, tremendous challenges already exist in predicting noisy stock prices. This paper proposes a hybrid model integrating the singular spectrum analysis (SSA) and the backpropagation neural network (BPNN) to forecast daily closing prices in stock markets. The model first decomposes the stock prices into several components using the SSA. Then, the extracted components are utilized for training BPNNs to forecast future prices. Compared with the BPNN, the hybrid SSA-BPNN model demonstrates a better predictive performance, indicating the SSA's ability to extract hidden information and reduce the noise effect of the original time series.
\end{abstract}

\section{INTRODUCTION}

The stock market is a public entity that provides financial activities to buy, sell, and issue shares of publicly traded companies [1]. A stock is a financial instrument that denotes ownership in a company and grants stockholders a portion of the company's assets and earnings. The company's stock is divided into shares at the initial public offering. Consequently, a share represents the smallest ownership unit in a particular company [2]. The initial price of a company's stock is determined by the company's value, revenues, and financial situation at the initial public offering. Afterward, its value fluctuates according to the stock's supply and demand. When a trader buys any number of shares of a company's stock, the expression "entered the market" is frequently used. If the price of the purchased stock moves, the actual worth of the investment changes. When the stock price increases, the trader will be in floating profit. In contrast, when the stock's value declines, the trader will be in floating loss. The final profit or loss is not realized until the trade is closed. Therefore, a trader must understand how stocks should be valued to purchase or sell stocks if their current value is less or greater than the fair value [2].

The stock market presents opportunities for individuals to invest in companies. The primary purpose of trading in stock markets is to profit from buying and selling listed stocks; however, it is considered a high-risk, high-yield investment [3]. In the last decade, soft computing models, e.g., artificial neural networks (ANN) and support vector machine (SVM) models, have shown to be effective tools for financial market prediction [4]. According to the works [5, 6], ANN models are the most commonly used to forecast various financial markets. $\mathrm{ANN}$ is a machine learning technique that simulates the human brain's structure and operation [7]. ANNs can precisely predict and distinguish nonlinear dataset patterns without prior knowledge, leading to their broad acceptability and adjustability [7-9]. Moreover, ANNs possess learning, generalization, and parallel processing features that efficiently resolve complex problems [10]. Consequently, ANNs are suitable for modeling time series characterized by significant fluctuations and discontinuities [11]. Unfortunately, most systems arising in practice, such as stock prices, are timevarying $[12,13]$ and contain much noise $[14,15]$, which reduces the ANNs' prediction efficiency. Consequently, data preprocessing techniques like singular spectrum analysis (SSA) are required to reduce the noise and extract interesting underlying information from the original time series $[16,17]$.

The SSA utilizes the singular value decomposition (SVD) to decompose time series and acquire a set of singular values that hold information about the original data [18-20]. The decomposed components are distinguished as a trend, periodic, or noise $[21,22]$. Hassani et al. [21] utilized the SSA to predict the British pound versus United states dollar daily exchange rate. The author concluded that the SSA model outperformed random walk models in forecasting exchange rate series. Menezes et al. explored the market linkages among the G7 countries via analyzing the stock market data based on SSA [23]. Wen et al. [19] introduced a hybrid model for predicting stock prices using SSA and SVM. The authors compared the predictive performance of the SSA-SVM to the single SVM model and discovered that the hybrid model outperforms the SVM prediction. Abdollahzade et al. [22] integrated the neurofuzzy models with SSA optimized by particle swarm to predict nonstationary chaotic time series. The proposed model was found to perform better in predicting nonlinear time series compared to various other methods. Lahmiri suggested a 
hybrid technique to predict intraday stock prices that combines SSA and SVR with particle swarm optimization (PSO) [18]. The suggested model's performance was validated in six intraday stock price series and demonstrated significant potential for analyzing and forecasting noisy time series. Xiao et al. combined the SSA and SVM to analyze and predict stock prices and applied them to the stock price of the Shanghai Stock Exchange (SSE) Composite Index [24]. Sulandari et al. presented a methodology for time series forecasting based on SSA and ANN and concluded that hybrid models outperformed single models [25].

In this study, a hybrid model integrating SSA and BPNN is developed to predict daily closing prices in stock markets. The model utilizes the SSA to decompose the stock prices to extract hidden information from the original time series and reduce the noise effect. For each decomposed component, a BPNN is constructed and trained to forecast one day ahead. The forecasted components are then aggregated to produce the final output. Unlike previous studies, the stock prices are first split into training and testing datasets; hence the testing set is hidden during the decomposition process. The proposed SSABPNN was validated using experimental data of the largest twenty market capital stocks listed in the Nasdaq stock exchange.

\section{RESEARCH METHODS}

\subsection{Singular spectrum analysis (SSA)}

The SSA has various applications, such as denoising signals, extracting the underlying trend, and forecasting applications [26]. The SSA approach comprises two interrelated stages, i.e., decomposition and reconstruction, and each comprises two steps [27]. The first stage involves decomposing the time series into simple and meaningful signals through embedding succeeded by SVD [18]. The second stage includes reconstructing the series for forecasting purposes via grouping and diagonal averaging [26]. This section summarizes the different stages and parameters selection criteria of the SSA.

\subsubsection{Decomposition}

The decomposition process is divided into two steps, which are embedding and SVD. Embedding is a preliminary step in analyzing time series that converts the original onedimensional series to a lagged multi-dimensional trajectory matrix [27]. For example, let $S_{t}=\left[S_{1}, S_{2}, \ldots, S_{N}\right]^{T}$ be a timeseries of length $N$, then the mapped trajectory matrix, with dimensions $L \times K$, is defined as [28, 29]:

$$
X=\left[X_{1}, X_{2}, \ldots, X_{K}\right]=\left(\begin{array}{cccc}
S_{1} & S_{2} & \cdots & S_{K} \\
S_{2} & S_{3} & \cdots & S_{K+1} \\
\vdots & \vdots & \ddots & \vdots \\
S_{L} & S_{L+1} & \cdots & S_{N}
\end{array}\right)
$$

where, $L$ is the window length representing the embedding dimension $(2 \leq L \leq N)$, and $K=N-L+1$. The trajectory matrix $X$ is a Hankel matrix since the elements along its antidiagonals are identical.

Following the embedding process, the SVD is utilized to factorize the trajectory matrix into biorthogonal elementary matrices [27]. This process is expressed as [30]:

$$
X=\sum_{r=1}^{R} X_{r}=U \Sigma V^{T}=\sum_{r=1}^{R} \sigma_{r} u_{r} v_{r}^{T}
$$

where, $X_{r}$ is the $r^{\text {th }}$ elementary matrix, $U$ and $V$ form an orthonormal system, $\Sigma$ is a diagonal matrix whose diagonal elements, $\sigma_{r}=\sqrt{\lambda_{r}}$, are the singular values of the lagcovariance matrix $X X^{T}, u_{r}$ denotes the eigenvector corresponding to the eigenvalue $\lambda_{r}, v_{r}$ represents the $r^{\text {th }}$ principal component, and $R$ is the rank of $X$. The set $\left(\sigma_{r}, u_{r}, v_{r}\right)$ is known as the $r^{\text {th }}$ eigentriple of the trajectory matrix.

\subsubsection{Reconstruction}

Reconstruction is the second stage of the SSA, in which the time-series is projected onto data-adaptive eigenvectors that reduce the dimensionality of the dataset via representing it in an optimal subspace [31]. The reconstruction process is divided into two steps, which are grouping and diagonal averaging. Grouping involves categorizing the elementary matrices, $X_{i}$, according to their eigentriples into groups. Then the matrices inside each group are summed up [26, 27]. Let $g$ $=\left\{i_{1}, i_{2}, \ldots, i_{r}\right\}$ be a group of $r$ selected eigentriples; hence, the matrix $X_{g}$ for group $g$ is expressed as:

$$
X_{g}=X_{i_{1}}+X_{i_{2}}+\cdots+X_{i_{r}}
$$

The increment of singular entropy due to eigentriple $i$ is represented by Eq. (4). The information within the time series is considered extracted when $\Delta E$ reaches an asymptotic value, and the following components are therefore caused by noise.

$$
\Delta E=-\left(\frac{\lambda_{i}}{\sum_{r=1}^{p} \lambda_{r}}\right) \log \left(\frac{\lambda_{i}}{\sum_{r=1}^{p} \lambda_{r}}\right)
$$

The second step in the reconstruction process is the diagonal averaging along the $N$ antidiagonal of the matrix $X_{g}$. This process transfers the matrix into a time series that will be a component of the original series $S_{t}$. The reconstructed time series will have a length $N$. Finally, the correlation coefficient between different components is utilized to determine their linear dependence. The correlation coefficient, $\rho$, in terms of the covariance of two components $X_{g_{1}}$ and $X_{g_{2}}$ is:

$$
\rho=\frac{\operatorname{cov}\left(X_{g_{1}}, X_{g_{2}}\right)}{\sigma_{1} \sigma_{2}}
$$

where, $\sigma$ is the component's standard deviations. In this work, components with $\rho \geq 0.4$ are aggregated.

\subsection{Backpropagation neural networks (BPNN)}

The artificial neural network comprises simple connected processors referred to as neurons, resembling the human brain's neurons. The neurons are joined by weighted connections that transfer signals between them. A neuron receives several input signals via its connections yet only produces one output signal. Then, the neuron's output signal is transferred via its outgoing connection into multiple branches that send identical signals. The outgoing branches terminate at other neurons' incoming connections [32]. The neuron's output 
is determined via an activation function that compares its calculated input to a predetermined threshold value.

There have been several investigations on activation functions in the literature, only a few have demonstrated their usefulness. For example, step and sign activation functions are frequently utilized in decision-making neurons to perform classification and pattern recognition tasks. On the other hand, the sigmoid function converts an input number between plus and minus infinity to a sensible value between zero and one. The linear activation function produces an output proportional to the neuron's weighted input. Generally, neurons with linear activation functions are frequently utilized to approximate linear functions [32].

A BPNN is a multilayer network with one or more hidden layers trained using a backpropagation learning algorithm (Figure 1). The network is typically composed of an input layer of source neurons, at least a hidden layer of computational neurons, and an output layer of computational neurons. The input signals are transmitted forward through successive layers. Each layer in a multilayer neural network serves a distinct purpose. The input layer receives signals from the external environment and distributes them to all neurons in the hidden layer. Indeed, because the input layer rarely contains computing neurons, it does not process input patterns. The hidden layer's neurons recognize the characteristics concealed in the input signals. Then, the output layer receives output signals from the hidden layer and establishes the output pattern for the entire network. The backpropagation learning algorithm is widely used for ANNs learning and is divided into two phases [32]. First, the network input layer is supplied with training data. After that, the network propagates the input data across each layer till the output layer generates the network's output. If this output does not match the required training data output, the error is measured and sent backward across the network from the output layer to the input layer. Weights are tuned in real-time as the error propagates.

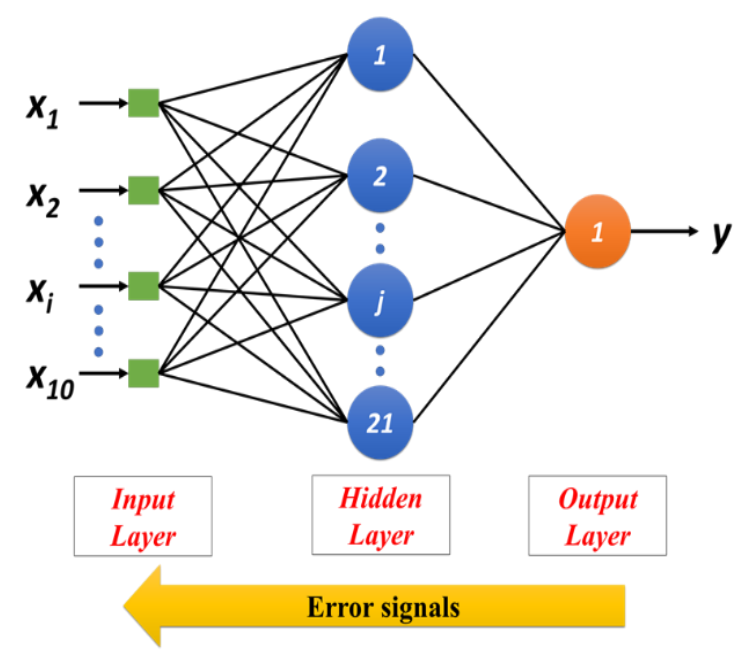

Figure 1. A three-layers BPNN

In this work, the daily closing prices are predicted by considering the ten preceding prices; hence there are ten input neurons in the input layer. According to Huang and Wang (2018), the number of the hidden neurons can be set approximately to be $2 \times n+1$, where $n$ is the number of the input neurons, and 1 denotes an output neuron [33]. Correspondingly, we adopt a $10 \times 21 \times 1$ structure of the BPNNs, as shown in Figure 1.

\subsection{The hybrid SSA-BPNN model}

This paper integrates the SSA and the BPNN to build a hybrid forecasting model, i.e., the SSA-BPNN model. First, the model utilizes the SSA to decompose the stock prices into several components. Then, the decomposed components are fed to the BPNNs to forecast one step of future daily prices. Finally, the forecasted outputs from the BPNNs are aggregated to produce the final prediction. Figure 2 shows a flow chart of the hybrid SSA-BPNN model, and the procedures are detailed as:

(1) Split the daily closing prices into a training dataset (70\%) and a testing dataset (30\%).

(2) Decompose the training dataset by the SSA using window length, $\mathrm{L}=14$

(3) Calculate the increment of singular entropy, $\Delta E$, of various singular values.

(4) Group elementary matrices when $\Delta E$ reaches an asymptotic value.

(5) Reconstruct time series components.

(6) Aggregate linearly dependent components $(\rho \geq 0.4)$.

(7) Normalize the reconstructed market components.

(8) Build and train three BPNNs $(10 \times 21 \times 1)$ to predict each market component.

(9) Forecast the future price of each point in the testing dataset using the following steps:

a) Decompose its prior prices as shown in steps $2-7$.

b) Forecast one step of each component.

c) Denormalize and aggregate the forecasted components.

d) Repeat steps (a) to (d) until all the testing points are predicted.

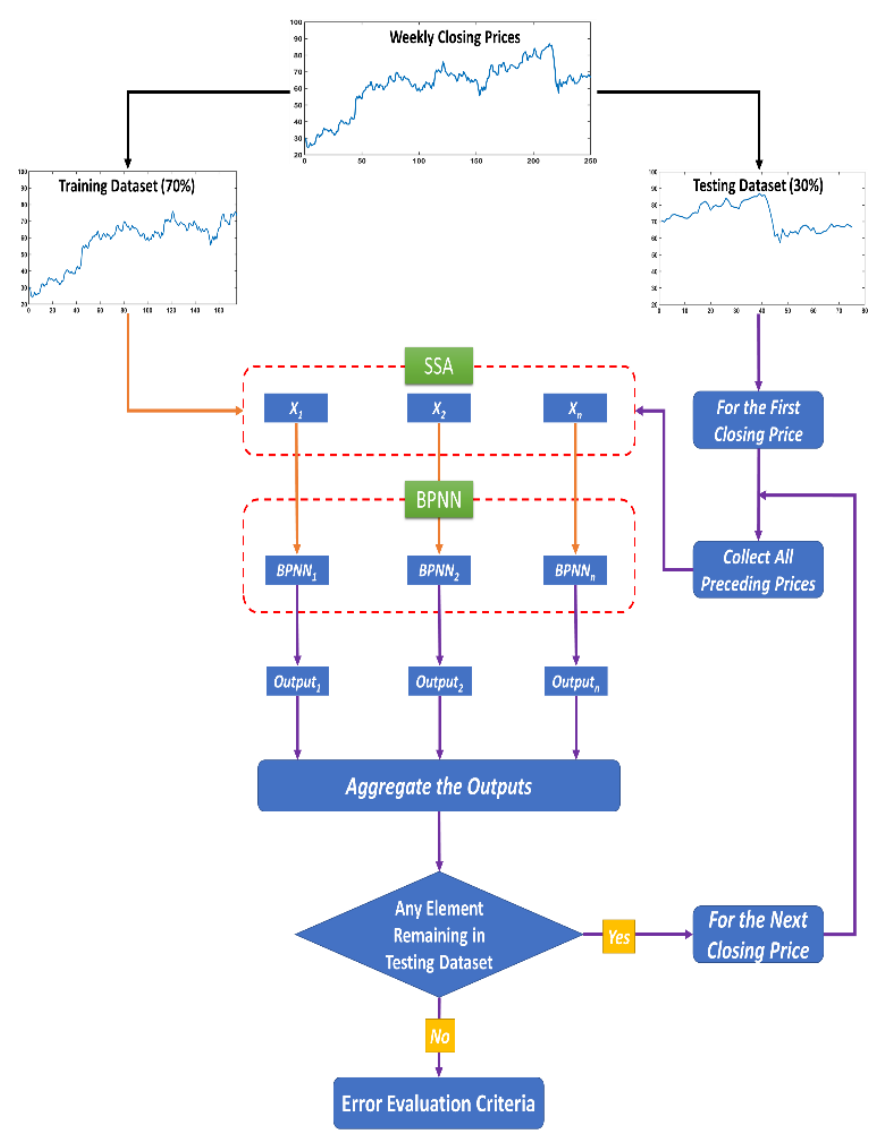

Figure 2. Flow chart of SSA-BPNN model 


\section{RESULTS AND DISCUSSION}

The data of the largest twenty market capital stocks listed in the Nasdaq stock exchange are utilized to validate the SSABPNN model. The data comprises the daily closing prices from January $2^{\text {nd }}, 2015$, to December $31^{\text {st }}, 2019$. There are 1007 trading days for each dataset. For illustration, the SSA decomposition process of the AAPL stock prices is detailed. The first $70 \%$ of the stock prices are chosen as the training set, while the remaining $30 \%$ comprise the testing dataset. The training series is decomposed into 14 elementary matrices, and the increment of singular entropy is calculated for each matrix. As shown in Figure 3, the increment of singular entropy saturates at the eighth order, and the difference in entropy between two consecutive orders is lower than $10^{-5}$. Therefore, elementary matrices from 8 to 14 are combined to form the noise term.

The next step is reconstructing the different matrices and calculating the correlation coefficient, as shown in Table 1. The correlation coefficient between reconstructed components one and two is -0.0013 , indicating that they are separable. Similarly, components 7 and 8 are separable. In contrast, components 2 through 7 have higher correlation coefficients and are combined in one component. Figure 4 displays the final reconstructed three components, i.e., RC1, RC2, and RC3, of the AAPL. RC1 represents the market trend. The medium oscillatory component $\mathrm{RC} 2$ is considered the market fluctuation, while the high oscillatory component RC3 indicates market noise.

Each reconstructed component is utilized for training a BPNN. Then the trained BPNNs are used to predict the various components. Finally, the forecasted components are aggregated to produce the forecasted prices. The mean average error (MAE), root mean square error (RMSE), and the mean absolute percentage error (MAPE) are employed to evaluate the prediction performances, as shown in Table 2.

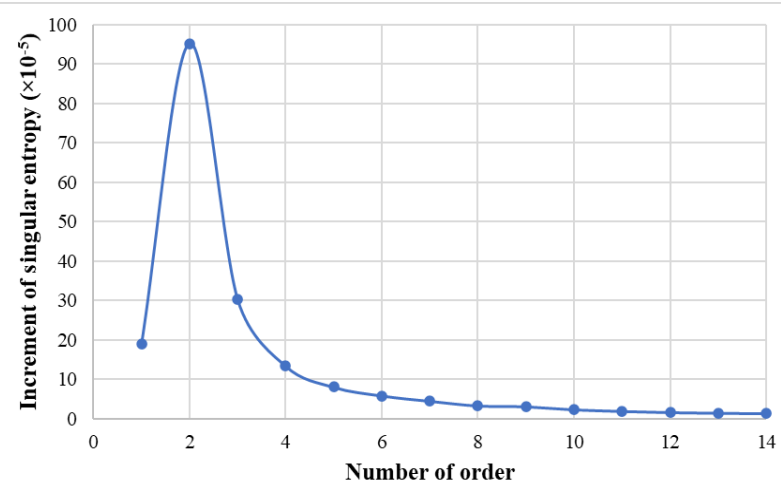

Figure 3. Increment of the singular entropy

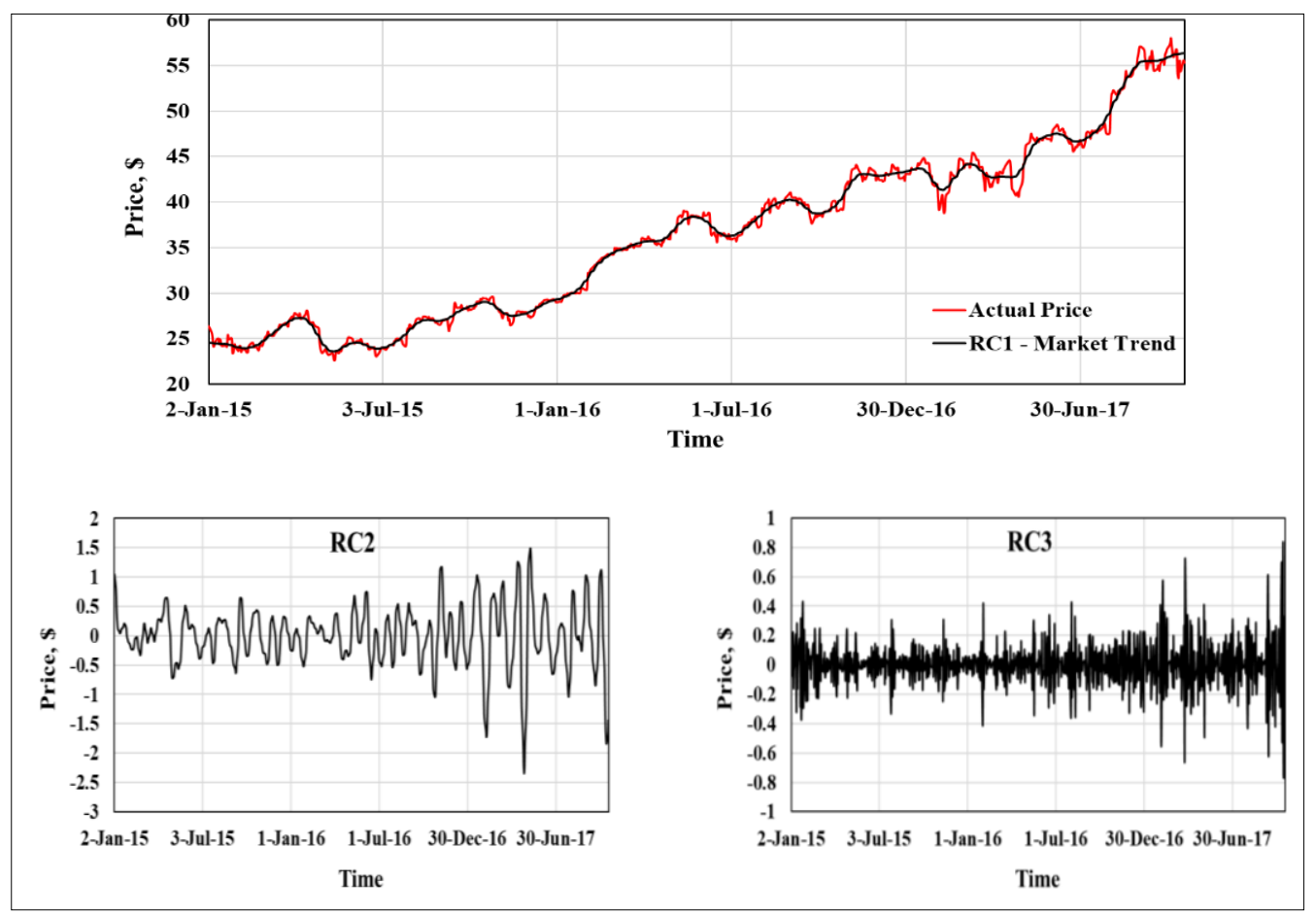

Figure 4. AAPL stock prices and decomposed components

Table 1. Correlation coefficients between the eight reconstructed components

\begin{tabular}{c|cccccccc}
\hline Reconstructed Matrix & $\mathbf{1}$ & $\mathbf{2}$ & $\mathbf{3}$ & $\mathbf{4}$ & $\mathbf{5}$ & $\mathbf{6}$ & $\mathbf{7}$ & $\mathbf{8}$ \\
\hline $\mathbf{1}$ & 1 & -0.0013 & -0.0112 & 0.0185 & -0.0066 & 0.0026 & -0.0076 & 0.0007 \\
$\mathbf{2}$ & -0.0013 & 1 & 0.6528 & 0.0479 & 0.0466 & 0.0092 & 0.0277 & 0.0052 \\
$\mathbf{3}$ & -0.0112 & 0.6528 & 1 & 0.5229 & 0.1686 & 0.0553 & 0.0286 & 0.0139 \\
$\mathbf{4}$ & 0.0185 & 0.0479 & 0.5229 & 1 & 0.5880 & 0.1234 & 0.0683 & 0.0261 \\
$\mathbf{5}$ & -0.0066 & 0.0466 & 0.1686 & 0.5880 & 1 & 0.5473 & 0.1646 & 0.0398 \\
$\mathbf{6}$ & 0.0026 & 0.0092 & 0.0553 & 0.1234 & 0.5473 & 1 & 0.6298 & 0.0807 \\
$\mathbf{7}$ & -0.0076 & 0.0277 & 0.0286 & 0.0683 & 0.1646 & 0.6298 & 1 & 0.2347 \\
$\mathbf{8}$ & 0.0007 & 0.0052 & 0.0139 & 0.0261 & 0.0398 & 0.0807 & 0.2347 & 1 \\
\hline
\end{tabular}


Table 2. Forecasting performance of the proposed SSA-BPNN and BPNN for individual stocks

\begin{tabular}{ccccccc}
\hline \multirow{2}{*}{ Stock } & \multicolumn{2}{c}{ MAE } & \multicolumn{2}{c}{ RMSE } & \multicolumn{2}{c}{ MAPE } \\
\cline { 2 - 7 } & BPNN & SSA-BPNN & BPNN & SSA-BPNN & BPNN & SSA-BPNN \\
\hline AAPL & 2.10 & $\mathbf{0 . 9 4}$ & 3.63 & $\mathbf{1 . 2 7}$ & 3.63 & $\mathbf{1 . 8 2}$ \\
MSFT & 10.64 & $\mathbf{3 . 2 5}$ & 13.98 & $\mathbf{4 . 2 5}$ & 7.70 & $\mathbf{2 . 4 4}$ \\
GOOG & 26.54 & $\mathbf{1 7 . 8 4}$ & 38.35 & $\mathbf{2 3 . 8 2}$ & 2.29 & $\mathbf{1 . 5 6}$ \\
GOOGL & 16.40 & $\mathbf{1 6 . 1 3}$ & 23.03 & $\mathbf{2 1 . 6 5}$ & 1.43 & $\mathbf{1 . 4 0}$ \\
AMZN & 36.34 & $\mathbf{2 8 . 4 5}$ & 58.23 & $\mathbf{4 0 . 2 8}$ & 2.13 & $\mathbf{1 . 6 7}$ \\
FB & 3.01 & $\mathbf{2 . 7 9}$ & 4.05 & $\mathbf{3 . 7 0}$ & 1.74 & $\mathbf{1 . 6 6}$ \\
TSLA & 1.64 & $\mathbf{1 . 5 6}$ & 2.37 & $\mathbf{2 . 1 7}$ & 2.91 & $\mathbf{2 . 7 6}$ \\
NVDA & 1.21 & $\mathbf{1 . 2 0}$ & 1.98 & $\mathbf{1 . 7 2}$ & 2.91 & $\mathbf{2 . 8 0}$ \\
ASML & 5.54 & $\mathbf{4 . 7 8}$ & 7.45 & $\mathbf{6 . 5 0}$ & 2.61 & $\mathbf{2 . 1 9}$ \\
ADI & 4.96 & $\mathbf{1 . 7 5}$ & 7.47 & $\mathbf{2 . 2 6}$ & 4.65 & $\mathbf{1 . 7 1}$ \\
PYPL & 3.87 & $\mathbf{3 . 1 9}$ & 5.24 & $\mathbf{4 . 0 0}$ & 3.71 & $\mathbf{3 . 0 4}$ \\
ADBE & 6.79 & $\mathbf{4 . 2 0}$ & 9.07 & $\mathbf{5 . 6 0}$ & 2.54 & $\mathbf{1 . 5 7}$ \\
CMCSA & 0.87 & $\mathbf{0 . 4 6}$ & 1.20 & $\mathbf{0 . 6 0}$ & 2.04 & $\mathbf{1 . 1 3}$ \\
NFLX & 7.68 & $\mathbf{7 . 4 5}$ & 10.25 & $\mathbf{9 . 7 8}$ & 2.47 & $\mathbf{2 . 3 6}$ \\
CSCO & 0.78 & $\mathbf{0 . 7 2}$ & 1.05 & $\mathbf{0 . 9 7}$ & 1.57 & $\mathbf{1 . 4 5}$ \\
INTC & 0.98 & $\mathbf{0 . 8 5}$ & 1.29 & $\mathbf{1 . 1 9}$ & 1.95 & $\mathbf{1 . 7 0}$ \\
PEP & 11.89 & $\mathbf{3 . 5 5}$ & 15.70 & $\mathbf{4 . 5 8}$ & 8.94 & $\mathbf{2 . 7 0}$ \\
COST & 31.48 & $\mathbf{9 . 8 0}$ & 44.51 & $\mathbf{1 2 . 9 7}$ & 11.06 & $\mathbf{3 . 6 1}$ \\
AVGO & 6.64 & $\mathbf{5 . 5 9}$ & 8.39 & $\mathbf{7 . 1 1}$ & 2.36 & $\mathbf{2 . 0 5}$ \\
TXN & 2.71 & $\mathbf{1 . 7 0}$ & 3.85 & $\mathbf{2 . 3 2}$ & 2.34 & $\mathbf{1 . 5 5}$ \\
\hline
\end{tabular}

Figure 5 displays a box plot for MAPE of BPNN and SSABPNN models. The SSA-BPNN model has the lowest median and mean MAPE for the twenty stocks involved in the analysis. Also, the MAPE for $75 \%$ of the stocks is lower than $2.8 \%$. Moreover, the hybrid model does not have any outliers.

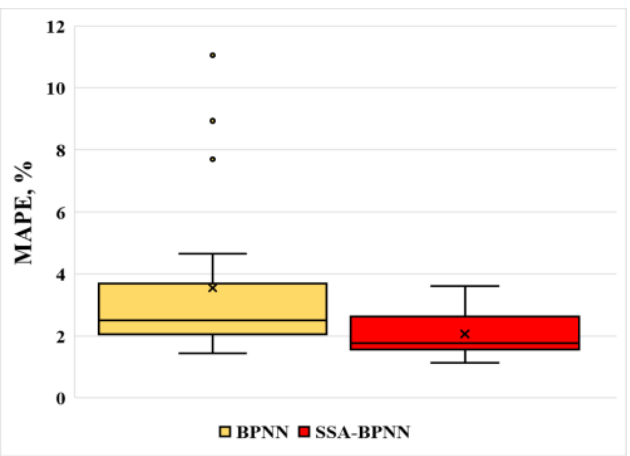

Figure 5. Box plot of MAPE for BPNN and SSA-BPNN models

From the previous analysis, it is evident that the proposed hybrid SSA-BPNN outperforms the single BPNN in terms of MAE, RMSE, and MAPE when predicting daily closing prices. The improved performance of the hybrid SSA-BPNN model is attributed to the SSA's ability to extract hidden information and reduce the noise effect in the original time series.

\section{CONCLUSION}

This paper proposed a hybrid forecasting model based on SSA and BPNN to predict daily closing prices in stock markets. The model employs the SSA to decompose the stock prices to reduce the noise and extract hidden information from the original price series. Also, BPNNs are built and trained to predict one day ahead of the different decomposed components. The BPNNs' outputs are summed up to produce the final forecasted prices.

The proposed model has been proven as an effective tool for predicting stock prices via conducting an empirical study with twenty stocks listed in the Nasdaq stock exchange. The research results indicated that the proposed SSA-BPNN model obtained the lowest MAE, MAPE, and RMSE for all stocks involved in the analysis. Furthermore, the model's superior forecasting ability is associated with its strength in capturing hidden information in financial time series, such as trends and volatility. Therefore, the presented approach is a promising tool for predicting daily closing prices.

\section{REFERENCES}

[1] Göçken, M., Özçalıcı, M., Boru, A., Dosdoğru, A.T. (2016). Integrating metaheuristics and artificial neural networks for improved stock price prediction. Expert Systems with Applications, 44: 320-331. https://doi.org/10.1016/j.eswa.2015.09.029

[2] Abirami, R., Vijaya, M.S. (2011). Stock price prediction using support vector regression. In International Conference on Computing and Communication Systems, pp. 588-597. https://doi.org/10.1007/978-3-642-292194_67

[3] Wang, Y., Xing, H. (2011). Time interval analysis on price prediction in stock market based on general regression neural networks. In International Conference on Electronic Commerce, Web Application, and Communication, pp. 160-166. http://dx.doi.org/10.1007/978-3-642-20370-1_27

[4] Kara, Y., Boyacioglu, M.A., Baykan, Ö.K. (2011). Predicting direction of stock price index movement using artificial neural networks and support vector machines: The sample of the Istanbul Stock Exchange. Expert systems with Applications, 38(5): 5311-5319. https://doi.org/10.1016/j.eswa.2010.10.027.

[5] Lin, X., Yang, Z., Song, Y. (2009). Short-term stock price prediction based on echo state networks. Expert systems with applications, 36(3): 7313-7317. https://doi.org/10.1016/j.eswa.2008.09.049

[6] Senapati, M.R., Das, S., Mishra, S. (2018). A novel model for stock price prediction using hybrid neural network. Journal of the Institution of Engineers (India): 
Series B $99(6)$

$555-563$

http://dx.doi.org/10.1007/s40031-018-0343-7

[7] Jothimani, D., Shankar, R., Yadav, S.S. (2015). A hybrid EMD-ANN model for stock price prediction. In International Conference on Swarm, Evolutionary, and Memetic Computing, pp. 60-70. http://dx.doi.org/10.1007/978-3-319-48959-9_6

[8] Büyükşahin, Ü.Ç., Ertekin, Ş. (2019). Improving forecasting accuracy of time series data using a new ARIMA-ANN hybrid method and empirical mode decomposition. Neurocomputing, 361: 151-163. http://dx.doi.org/10.1016/j.neucom.2019.05.099

[9] Safari, A., Davallou, M. (2018). Oil price forecasting using a hybrid model. Energy, 148: 49-58. http://dx.doi.org/10.1016/j.energy.2018.01.007

[10] Wang, J.Z., Wang, J.J., Zhang, Z.G., Guo, S.P. (2011). Forecasting stock indices with back propagation neural network. Expert Systems with Applications, 38(11): 14346-14355. https://doi.org/10.1016/j.eswa.2011.04.222

[11] Ruiz, L.G.B., Cuéllar, M.P., Calvo-Flores, M.D., Jiménez, M.D.C.P. (2016). An application of non-linear autoregressive neural networks to predict energy consumption in public buildings. Energies, 9(9): 684. https://doi.org/10.3390/en9090684

[12] Abdelaziz, T.H. (2018). Stabilization of linear timevarying systems using proportional-derivative state feedback. Transactions of the Institute of Measurement and Control, 40(7): 2100-2115. https://doi.org/10.1177/0142331217697787

[13] Abdelaziz, T.H. (2016). Eigenstructure assignment by displacement-acceleration feedback for second-order systems. Journal of Dynamic Systems, Measurement, and Control, 138(6). https://doi.org/10.1115/1.4032877

[14] Wei, L.Y. (2016). A hybrid ANFIS model based on empirical mode decomposition for stock time series forecasting. Applied Soft Computing, 42: 368-376. https://doi.org/10.1016/j.asoc.2016.01.027

[15] Lu, C.J., Chiu, C.C., Yang, J.L. (2009). Integrating nonlinear independent component analysis and neural network in stock price prediction. In International Conference on Industrial, Engineering and Other Applications of Applied Intelligent Systems, pp. 614-623. https://doi.org/10.1007/978-3-642-02568-6_62

[16] Lu, C.J. (2013). Hybridizing nonlinear independent component analysis and support vector regression with particle swarm optimization for stock index forecasting. Neural Computing and Applications, 23(7): 2417-2427. https://doi.org/10.1007/s00521-012-1198-5

[17] Kao, L.J., Chiu, C.C., Lu, C.J., Yang, J.L. (2013). Integration of nonlinear independent component analysis and support vector regression for stock price forecasting. Neurocomputing, 99: 534-542. https://doi.org/10.1016/j.neucom.2012.06.037

[18] Lahmiri, S. (2018). Minute-ahead stock price forecasting based on singular spectrum analysis and support vector regression. Applied Mathematics and Computation, 320: 444-451. https://doi.org/10.1016/j.amc.2017.09.049

[19] Wen, F.H., Xiao, J.H., He, Z. F., Gong, X. (2014). Stock price prediction based on SSA and SVM. Procedia Computer Science, 31: 625-631. https://doi.org/10.1016/j.procs.2014.05.309

[20] Trendafilova, I. (2021). Singular spectrum analysis for the investigation of structural vibrations. Engineering
Structures,

242:

112531.

https://doi.org/10.1016/j.engstruct.2021.112531

[21] Hassani, H., Soofi, A.S., Zhigljavsky, A.A. (2010). Predicting daily exchange rate with singular spectrum analysis. Nonlinear Analysis: Real World Applications, 11(3): https://doi.org/10.1016/j.nonrwa.2009.05.008 2023-2034.

[22] Abdollahzade, M., Miranian, A., Hassani, H., Iranmanesh, H. (2015). A new hybrid enhanced local linear neuro-fuzzy model based on the optimized singular spectrum analysis and its application for nonlinear and chaotic time series forecasting. Information Sciences, 295: 107-125. https://doi.org/10.1016/j.ins.2014.09.002

[23] Menezes, R., Dionísio, A., Hassani, H. (2012). On the globalization of stock markets: An application of vector error correction model, mutual information and singular spectrum analysis to the G7 countries. The Quarterly Review of Economics and Finance, 52(4): 369-384. https://doi.org/10.1016/j.qref.2012.10.002

[24] Xiao, J., Zhu, X., Huang, C., Yang, X., Wen, F., Zhong, M. (2019). A new approach for stock price analysis and prediction based on SSA and SVM. International Journal of Information Technology \& Decision Making, 18(1): 287-310. https://doi.org/10.1142/S021962201841002X

[25] Sulandari, W., Subanar, S., Lee, M.H., Rodrigues, P.C. (2020). Time series forecasting using singular spectrum analysis, fuzzy systems and neural networks. MethodsX, 7: 101015. https://doi.org/10.1016/j.mex.2020.101015

[26] Lin, Y., Ling, B.W.K., Xu, N., Lam, R.W.K., Ho, C.Y.F. (2020). Effectiveness analysis of bio-electronic stimulation therapy to Parkinson's diseases via joint singular spectrum analysis and discrete fourier transform approach. Biomedical Signal Processing and Control, 62: 102131. https://doi.org/10.1016/j.bspc.2020.102131

[27] Hassani, H. (2007). Singular spectrum analysis: Methodology and comparison. Journal of Data Sciences, 5: 239-257. https://doi.org/10.6339/JDS.2007.05(2).396

[28] Rocco S.C.M. (2013). Singular spectrum analysis and forecasting of failure time series. Reliability Engineering and System Safety, 114: 126-136. https://doi.org/10.1016/j.ress.2013.01.007

[29] De Carvalho, M., Rua, A. (2017). Real-time nowcasting the US output gap: Singular spectrum analysis at work. International Journal of Forecasting, 33(1): 185-198. https://doi.org/10.1016/j.ijforecast.2015.09.004

[30] Leles, M.C., Sansão, J.P.H., Mozelli, L.A., Guimarães, H.N. (2018). Improving reconstruction of time-series based in Singular Spectrum Analysis: A segmentation approach. Digital Signal Processing, 77: 63-76. https://doi.org/10.1016/j.dsp.2017.10.025

[31] Groth, A., Ghil, M. (2015). Monte Carlo singular spectrum analysis (SSA) revisited: Detecting oscillator clusters in multivariate datasets. Journal of Climate, 28(19): 7873-7893. https://doi.org/10.1175/JCLI-D-150100.1

[32] Negnevitsky, M. (2005). Artificial Intelligence: A Guide to Intelligent Systems. Pearson education.

[33] Huang, L., Wang, J. (2018). Forecasting energy fluctuation model by wavelet decomposition and stochastic recurrent wavelet neural network. Neurocomputing, 309, 70-82. https://doi.org/10.1016/j.neucom.2018.04.071 